\title{
Etude sur modèle réduit de la passe à poissons du barrage de Bergerac sur la Dordogne
}

\author{
Hydraulic model studies for Bergerac dam fishway \\ on the Dordogne river
}

\author{
M. Larinier \\ CEMAGREF \\ Castanet Tolosan
}

\author{
D. Trivellato \\ Institut de Mécanique des fluides \\ de Toulouse
}

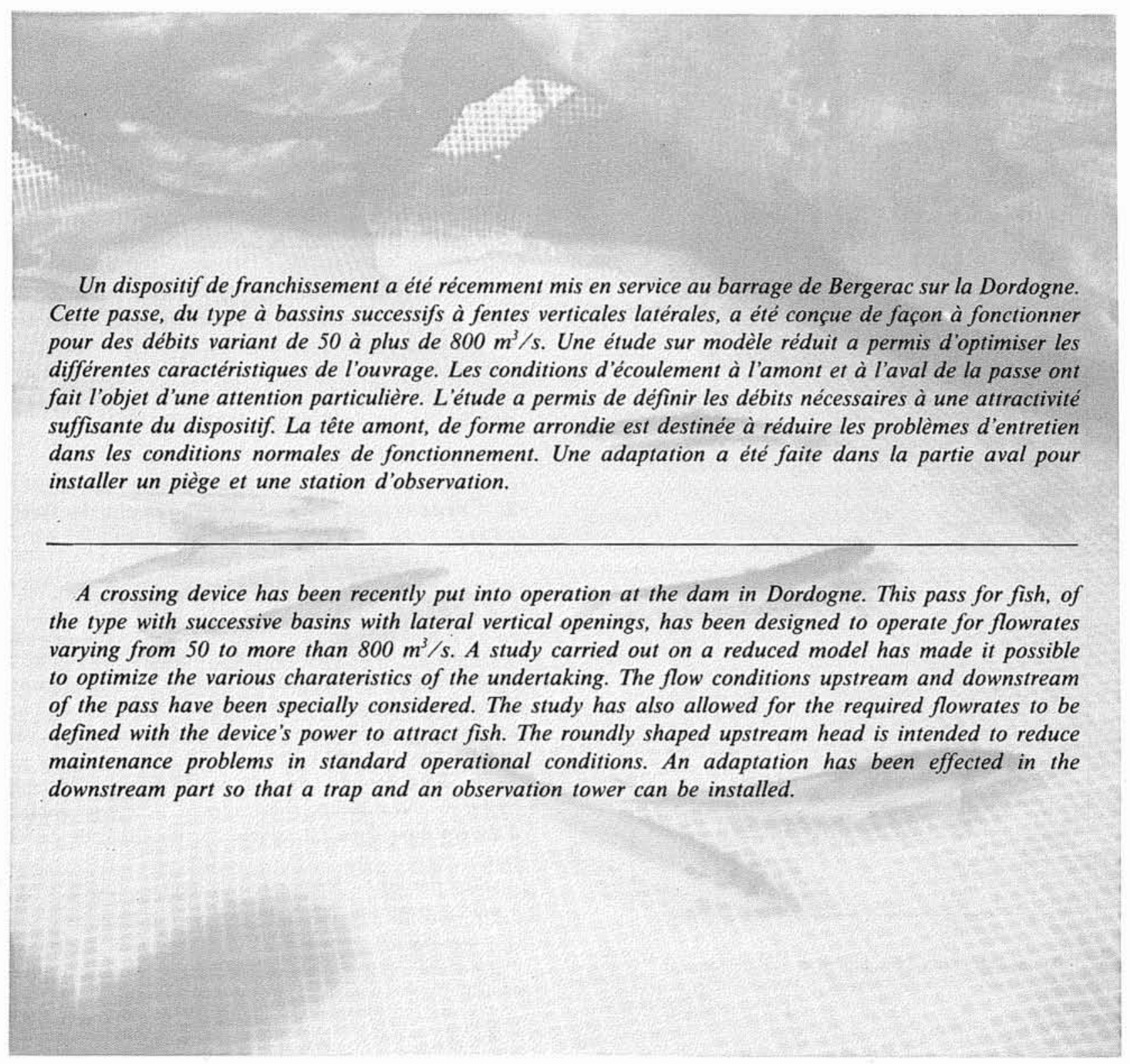




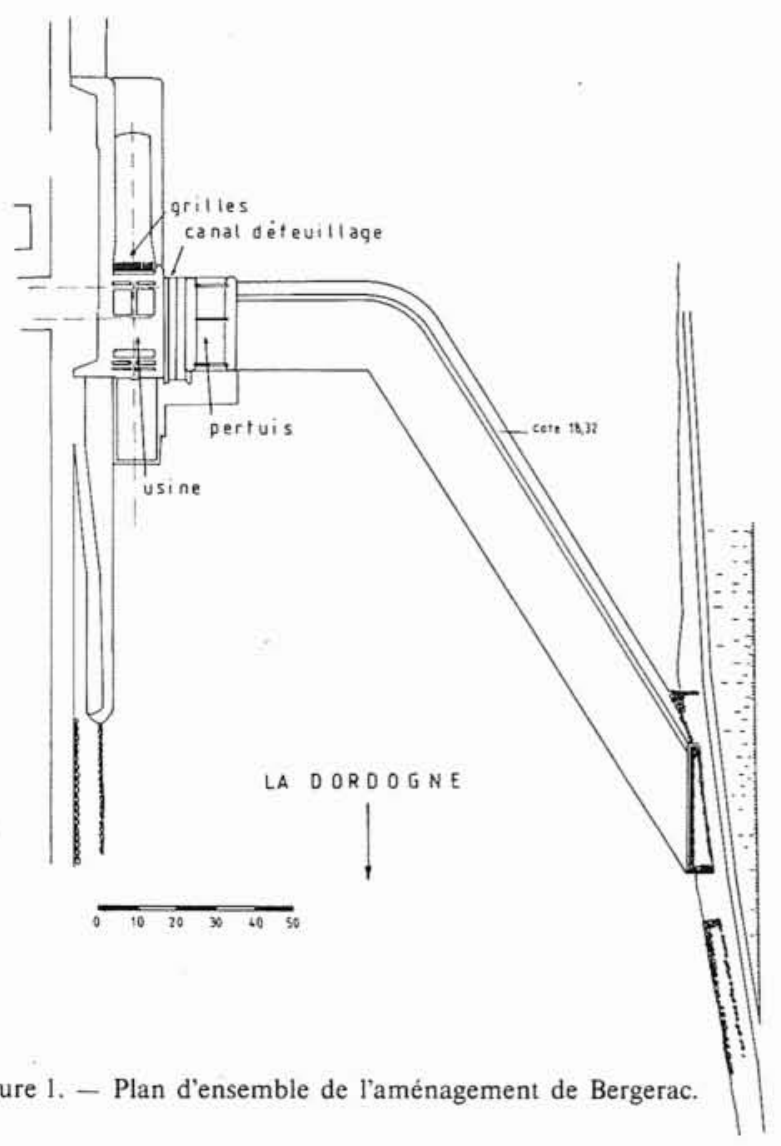

Figure 1. - Plan d'ensemble de l'aménagement de Bergerac.

\section{Introduction}

Le saumon est présent dans tout le bassin de la Dordogne jusqu'au milieu du siècle dernier. Dès la construction des barrages de Bergerac et Mauzac, et malgré l'aménagement de plusieurs dispositifs de franchissement ${ }^{(1)}$, la pêche au saumon et à l'alose demeure pratiquement cantonnée à l'aval de Bergerac. La construction et la mise en service de l'aménagement de Tuilière vers 1906 ne fait qu'aggraver la situation en interdisant l'accès des migrateurs aux frayères situées plus en amont. Les pouvoirs publics, conscients de la gravité de la situation, décident dès 1885 la création d'une pisciculture en aval du barrage de Bergerac. Les opérations de repeuplement se soldent cependant par un échec ${ }^{(2)}$ et les alevinages cessent vers 1930. A ces différents facteurs, il convient d'ajouter la pollution de la Poudrerie nationale de Bergerac qui avait augmenté considérablement sa production vers 1917-1918 (Roule, 1929) et déversait directement dans la rivière ses eaux résiduaires. Le nombre de captures de saumons en aval de Bergerac passe de plusieurs centaines vers 1900-1915 à quelques individus après 1923 .

Un plan de restauration du saumon atlantique et de protection des autres espèces migratrices a été lancé depuis une dizaine d'années sur la Dordogne, les premiers essais de repeuplement ayant débuté vers 1978. Dumas (1979) et Pustelnik (1985) évaluent à 2 000-5 000 adultes le potentiel de production en saumons du bassin de la Dordogne entre les barrages de Mauzac et d'Argentat. La réussite de ce plan passe par la construction d'ouvrages de franchissement efficaces aux trois barrages du Bergeracois.

L'étude et la construction de la passe située sur l'ouvrage le plus aval, objet de la présente communication, ont été financées conjointement par E.D.F. et le ministère de l'Environnement. L'étude sur modèle réduit considérée comme indispensable a été réalisée à l'Institut de mécanique des fluides de Toulouse (I.M.F.T., 1983).

\section{Caractéristiques de l'aménagement de Berge- rac}

L'aménagement de Bergerac (figure 1) est constitué, de la rive droite à la rive gauche :

- d'une usine hydroélectrique, installée dans une ancienne écluse de navigation, équipée de deux groupes bulbes monoblocs à pales et directrices fixes. Chaque groupe peut absorber un débit de $28,6 \mathrm{~m}^{3} / \mathrm{s}$. La puissance installée est de $1450 \mathrm{~kW}$;

- de deux couloirs de 1,80 m de largeur, anciennes passes à ralentisseurs construites il y a une trentaine d'années dont l'une est utilisée comme canal de défeuillage;

- d'un pertuis de près de $7 \mathrm{~m}$ de largeur, ancienne passe à sauter construite vers 1887 ;

(1) Dans un rappoit de 1897, Philippe signale déjà l'existence de quatre passes à poissons au barrage de Bergerac.

(2) Les transports de poissons vivants posent à cette époque de gros problèmes techniques et les déversements sont effectués dans des zones convenant mal aux juvéniles (Dumas, 1979).

Figure 2. - Relations cotes plan d'eau amont et aval en fonction du débit. 
- d'un déversoir de $165 \mathrm{~m}$ dont la crête est arasée à la cote 18,32 NGF.

La chute est nettement sous équipée compte tenu du régime hydrologique de la Dordogne, dont le débit moyen annuel est voisin de $280 \mathrm{~m}^{3} / \mathrm{s}$. Les débits moyens mensuels varient de $100 \mathrm{~m}^{3} / \mathrm{s}$ environ (juillet-août) à plus de 450 $\mathrm{m}^{3} / \mathrm{s}$ (décembre-janvier-février). Les débits d'étiage descendent rarement au-dessous de $30 \mathrm{~m}^{3} / \mathrm{s}$. La chute au barrage (figure 2) varie de $4,20 \mathrm{~m}$ en étiage à moins de 2 mètres pour un débit en Dordogne voisin de $1000 \mathrm{~m}^{3} / \mathrm{s}$.

L'usine est arrêtée lorsque le débit en Dordogne dépasse $600 \mathrm{~m}^{3} / \mathrm{s}$.

La dénivellation pour les faibles débits et la présence d'un ressaut très violent pour les débits plus élevés rendent le barrage très difficilement franchissable pour la plupart des espèces migratrices.

A notre connaissance, sept passes plus ou moins sommaires ont été aménagées au barrage de Bergerac depuis sa construction : deux passes à chicanes, une passe " à sauter " dans le pertuis de $6,40 \mathrm{~m}$ de lárgeur, une passe en bois de type Caméré, deux passes à ralentisseurs de type PIRO-PLAN et une " écharpe".

Le manque d'efficacité de ces différents dispositifs peut être attribué à plusieurs causes :

- soit à leur manque d'attractivité (débit transitant dans la passe trop faible eu égard au débit de la Dordogne, mauvaise implantation);

- soit à leur inaptitude à s'adapter aux conditions de niveaux amont et aval très variables;

- soit à leur conception même (passes trop vulnérables aux crues comme la passe Caméré ou ne s'adaptant pas à des migrateurs comme l'alose, comme la passe "à sauter " ou "l'écharpe ».

\section{Choix du type et de l'implantation de la passe à poissons}

Le dispositif de franchissement est destiné en priorité aux grands migrateurs - principalement le saumon, la truite de mer, l'alose et la lamproie - et accessoirement aux migrateurs holobiotiques. Les périodes de migration de ces différentes espèces recouvrent pratiquement toute l'année à l'exception de deux mois (août et septembre). Les conditions de débit - et partant de niveaux amont et aval - pour lesquelles la passe doit rester efficace sont donc très variables. L'examen des courbes de débits classés a permis de fixer comme plage de fonctionnement 50-800 $\mathrm{m}^{3} / \mathrm{s}$, ce qui correspond à des variations des niveaux d'eau amont et aval du barrage de près de 2,00 et 4,00 mètres respectivement.

Seuls un ascenseur ou une passe à bassins successifs à fentes verticales peuvent s'adapter aux fluctuations de niveaux et aux différentes espèces migratrices en présence. Le souci de réduire au maximum les contraintes inhérentes au fonctionnement et à la maintenance du dispositif a orienté le choix vers la passe à bassins successifs.

Le pertuis de l'ancienne passe « à sauter ", de par sa situation entre l'usine et l'angle amont du déversoir, semblait a priori l'endroit le plus favorable pour l'implantation du dispositif de franchissement.
Le débit dans la passe a été fixé à $2,5 \mathrm{~m}^{3} / \mathrm{s}$ pour un plan d'eau amont à 18,32 NGF, correspondant aux débits d'étiage. La largeur et la longueur des bassins ont été fixées respectivement à 6,00 et $4,50 \mathrm{~m}$; le dénivelé entre deux bassins successifs a été limité à $0,30 \mathrm{~m}$, la puissance dissipée théorique par unité de volume dans les bassins à $150 \mathrm{~W} / \mathrm{m}^{3}$, ce qui détermine le volume minimum d'eau dans les bassins en étiage (environ $50 \mathrm{~m}^{3}$ ).

La largeur minimale des fentes a été fixée à $0,45 \mathrm{~m}$, compte tenu de la présence de l'alose (Larinier, Travade, 1982).

L'étude sur modèle est destinée :

- à préciser le calage de la passe, à s'assurer que les critères sur les puissances dissipées et les chutes sont respectés;

- à positionner l'entrée - entrée pour le poisson, donc partie aval - et à étudier l'attrait de la passe dans le bief aval;

- à définir le débit complémentaire d'attrait nécessaire et à donner les caractéristiques d'un ouvrage permettant sa prise, la dissipation de son énergie et son adjonction dans la partie aval de la passe;

- à étudier une forme de tête amont de façon à réduire l'entraînement des corps dérivants dans la passe.

\section{Calage de la passe et écoulement dans les bassins}

Une première étude a été réalisée en canal vitré sur cinq cellules identiques afin d'examiner l'écoulement dans les bassins et de caler la passe.

La largeur des fentes a été portée à $0,55 \mathrm{~m}$, de façon à faciliter le passage des aloses. La relation hauteur d'eau-débit (figure 3) en régime pseudo-uniforme, c'est-à-dire lorsque la profondeur d'eau moyenne reste à peu près constante d'un bassin à l'autre, a permis le calage de la passe pour le débit de projet $\left(2,5 \mathrm{~m}^{3} / \mathrm{s}\right)$ : dans chaque fente a été placé un seuil dont l'arête horizontale est arasée à $0,80 \mathrm{~m}$ au-dessus du radier. Un orifice de $0,15 \mathrm{~m}$ a été aménagé dans la partie inférieure du seuil.

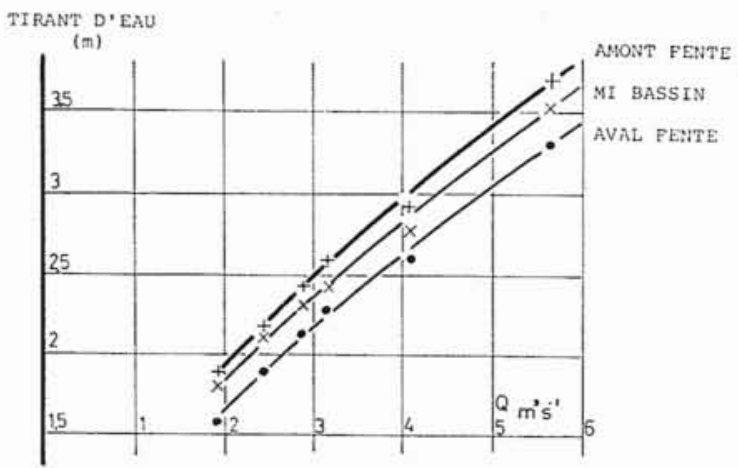

Figure 3. - Relation tirant d'eau-débit en écoulement pseudouniforme dans les bassins. 


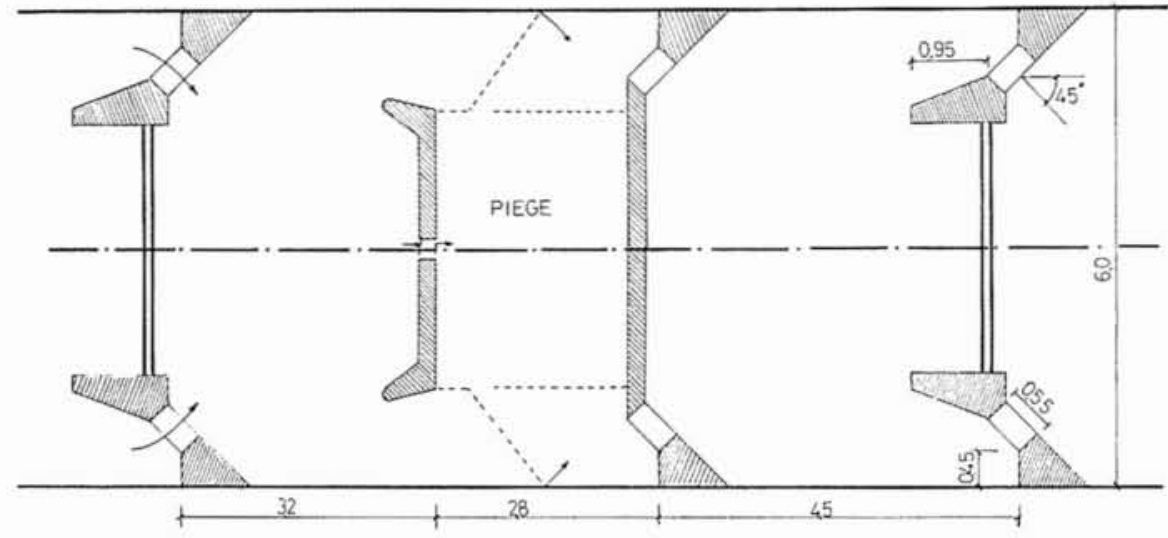

Figure 4. - Caractéristiques des fentes et du piège.

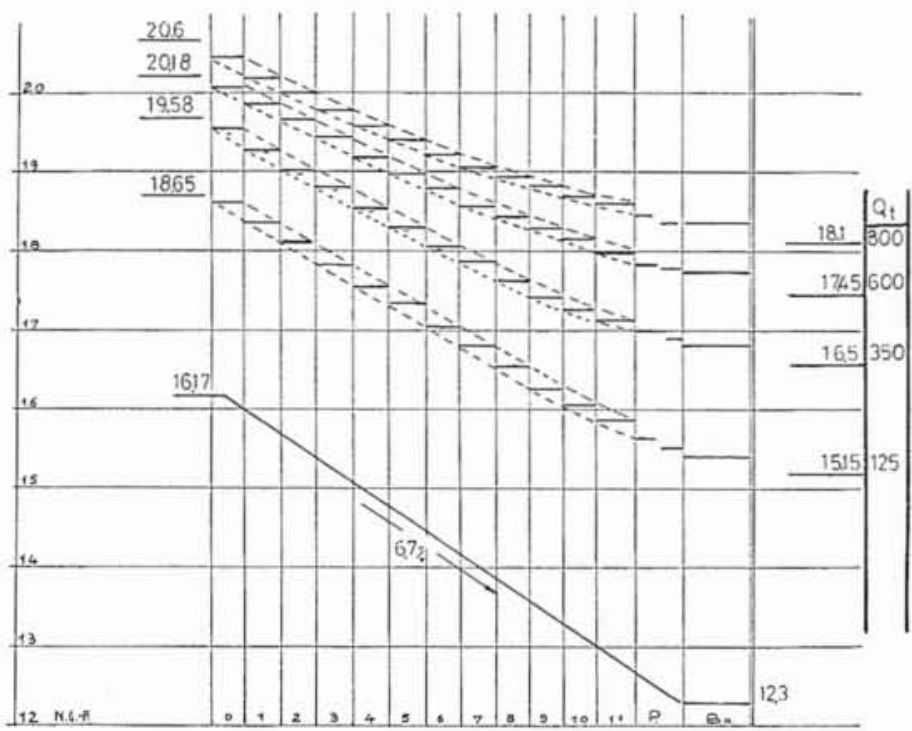

Figure 5. - Évolution du profil en long de l'écoulement dans la passe en fonction du débit en Dordogne.

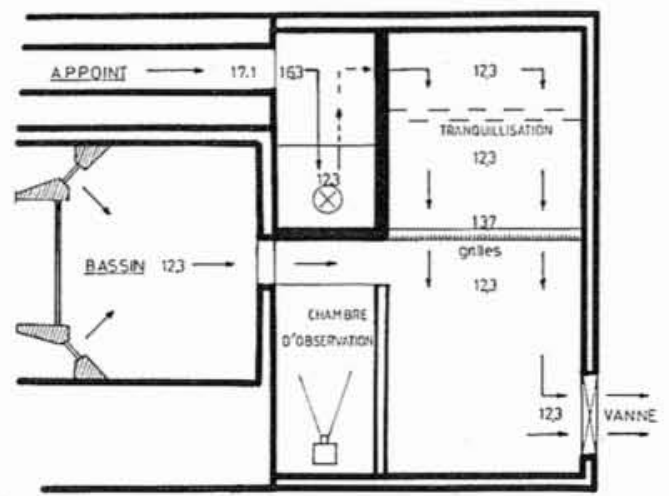

Figure 6: Bassin aval de la passe et dispositif d'adjonction du débit complémentaire d'attrait.
Les caractéristiques des fentes (figure 4) sont très voisines de celles adoptées pour la passe de Belleville (I.M.F.T., 1982); toutefois, la partie aval des piles latérales a été modifiée de façon à supprimer tout décrochement brusque et à assurer ainsi un meilleur guidage des migrateurs (risque de piégeage des aloses dans les encoignures).

L'écoulement dans les bassins (Photo 1) est relativement complexe, l'interaction des deux jets issus des fentes amont donnant des structures très instables qui génèrent des fluctuations de vitesses importantes au débouché des fentes suivantes. Des mesures de temps de séjour et de transit par injection de traceur ont permis une meilleure approche du phénomène de brassage de l'eau dans les bassins.

\section{Le modèle général: Implantation et écoule- ment dans la passe}

Le modèle général (Photo 2) reproduit, à l'échelle 1/22:

- l'usine : un dispositif permet de régler et mesurer le débit absorbé et restitué à l'aval;

- le canal de défeuillage:

- la passe à poissons avec ses ouvrages amont et aval;

- la partie rectiligne normale à l'axe longitudinal du seuil déversant, cette partie ayant été prolongée et portée à une longueur de $45 \mathrm{~m}$.

Les conditions aux limites du modèle sont des profils en travers de la Dordogne situés à $90 \mathrm{~m}$ en amont et à 220 mètres en aval du barrage.

La passe est constituée d'une série de 12 bassins dont les caractéristiques ont été définies sur le modèle partiel. Le radier au niveau de la fente amont est calé à la cote 16,00 NGF, l'extrémité aval de ce radier étant à la cote 12,30 NGF.

Une adaptation (figure 4) a été faite dans l'un des bassins ${ }^{(3)}$ pour installer un piège destiné à contrôler l'efficacité de la passe et permettre la capture et le transport de géniteurs. Le piège consiste en une chambre 


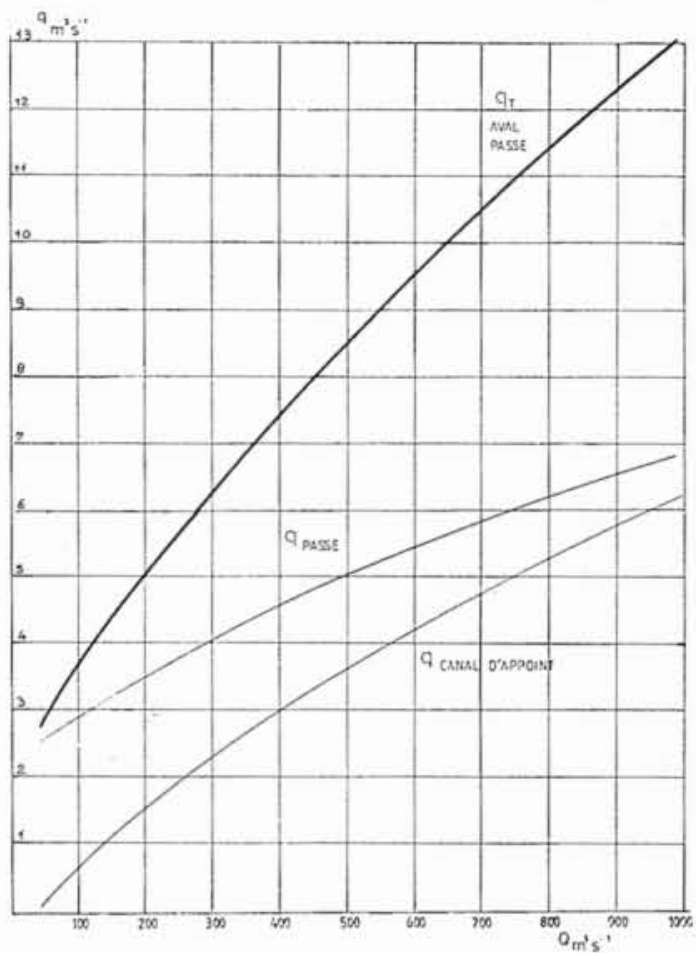

Figure 7. - Débit aux ouvrages.

Photo 3. - Attrait de la passe dans le bief aval pour un débit en Dordogne de $125 \mathrm{~m}^{3} / \mathrm{s}$.

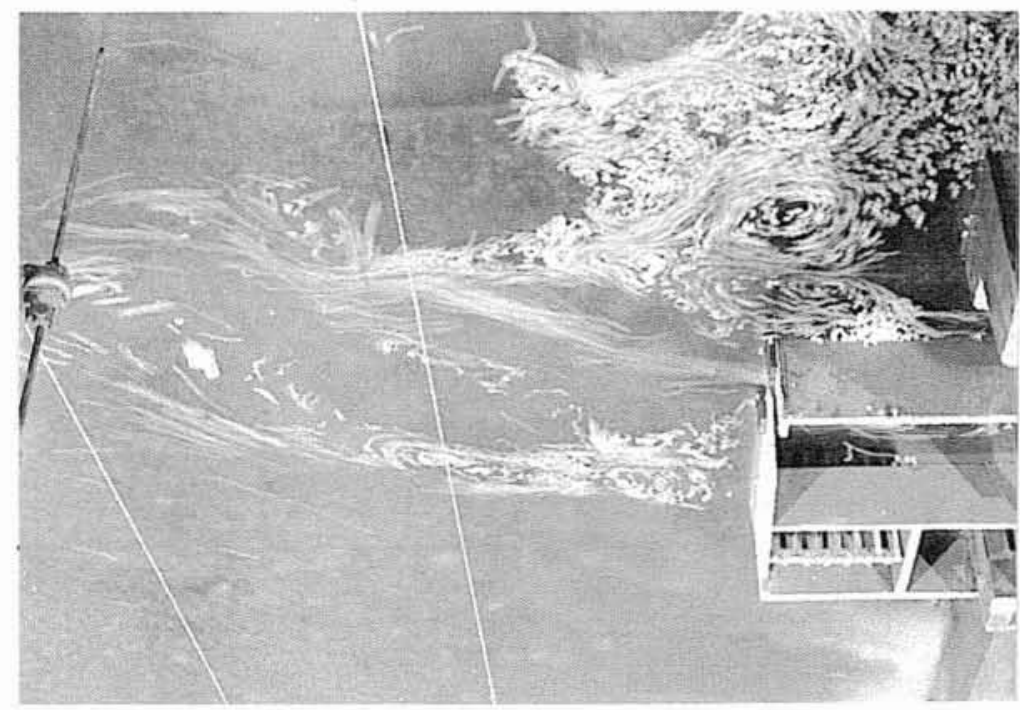

Photo 4. - Écoulement au niveau de la tête amont de l'ouvrage.

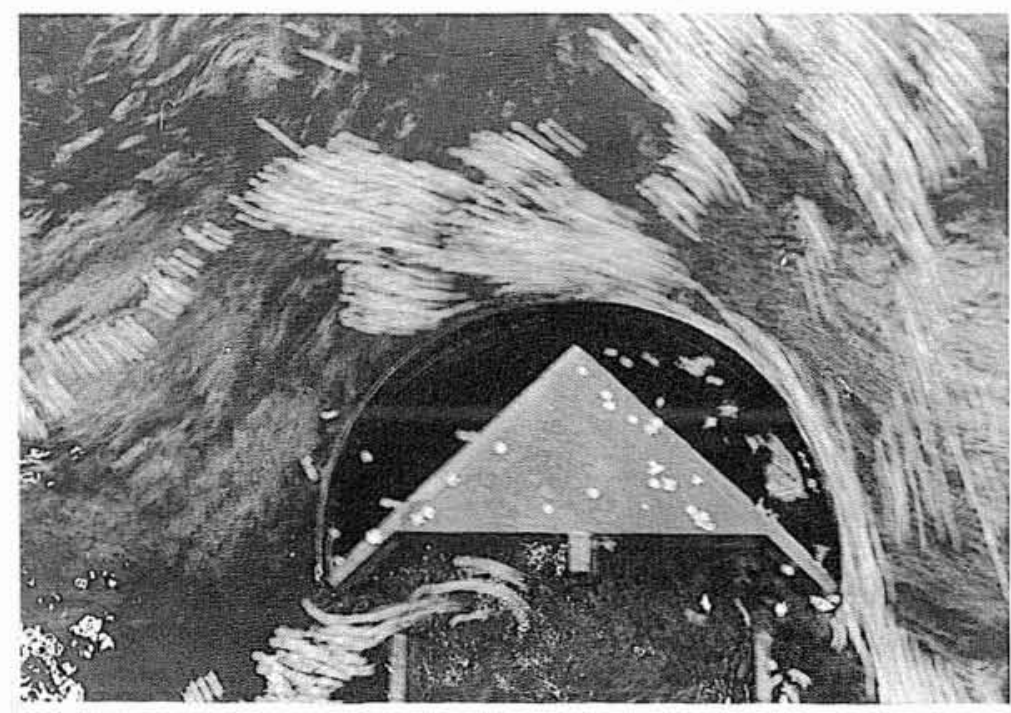

Un canal de 1 mètre de largeur situé dans la pile rive gauche permet l'introduction du débit complémentaire d'attrait. La prise d'eau est protégée par un masque au bordé inférieur calé à la cote 17,82. La dissipation d'énergie du débit d'appoint s'effectue dans trois bassins successifs (Fig. 6). Ce débit est injecté après tranquillisation dans le bassin aval à travers une ouverture de $5 \mathrm{~m} \times$ $3,4 \mathrm{~m}$ équipée de grilles. Ce bassin communique avec le bief aval par une ouverture de 2 mètres de largeur qui constitue l'entrée de la passe. Elle est munie d'une vanne assujettie à un système de réglage maintenant une dénivellation constante entre les cotes des plans d'eau situés de part et d'autre de cette vanne (de l'ordre de 0,25-0,30 m).

Le débit total (passe + appoint) varie de $2,5 \mathrm{~m}^{3} / \mathrm{s}$ environ à plus de $11 \mathrm{~m}^{3} / \mathrm{s}$ pour un débit de $800 \mathrm{~m}^{3} / \mathrm{s}$ en Dordogne (Fig. 7).

Le débit issu de la passe constitue quelles que soient les conditions aval (structures organisées en aval de l'usine, ressaut ou veine ondulée au pied de la chaussée) un jet bien constitué, homogène. La vanne permet d'obtenir des vitesses de $2,50 \mathrm{~m} / \mathrm{s}$ à l'entrée et supérieures à $1 \mathrm{~m} / \mathrm{s}$ à plus de 10 mètres de l'ouvrage. Ce jet s'oriente le plus près possible de l'écoulement principal, c'est-à-dire le long du canal de fuite tant que l'usine fonctionne (Photo 3) et en direction de l'écoulement principal lorsque les débits déversés augmentent. L'ouverture du canal de défeuillage (Fig. 8) anihile l'influence d'un vaste courant de retour qui viendrait rabattre le jet sous l'écoulement principal du seuil.

\section{7. Écoulement dans le bief amont et protection de la passe}

L'alimentation de la passe s'effectue par deux prises latérales de 1,30 m de largeur protégées par un avant bec. Initialement, la tête amont était formée d'un dièdre constitué de 2 plans verticaux. Cette configuration a été abandonnée, son efficacité de protection contre les corps dérivants étant faible. L'écoulement dans le bief amont présente fréquemment un angle avec la passe suivant les conditions de fonctionnement de l'usine et l'importance des débits déversés. Les décollements aux angles vifs provoquent de vastes courants de retour qui ramènent les corps flottants le long de la passe jusqu'aux pertuis d'alimentation.

Une tête arrondie de 9 mètres de diamètre a été retenue. Cette forme réduit considérablement les décollements des filets liquides.

Tant que l'usine absorbe la plus grande partie du débit du cours d'eau, il existe une différence de niveau de part et d'autre de la passe. Un très léger courant de retour se forme sur le flanc gauche de la passe et une zone d'eau morte sur le flanc droit. L'alimentation du bassin amont de la passe est correcte et la très légère inégalité aux prises d'eau est réduite au niveau des fentes amont (photo 4). Lorsque tout le débit déverse sur la chaussée, le courant de retour qui se formerait sur le flanc droit de la passe est supprimé par l'ouverture du canal de défeuillage (fig. 8). Le débit est inégalement réparti aux prises d'eau, mais sans conséquences graves. 


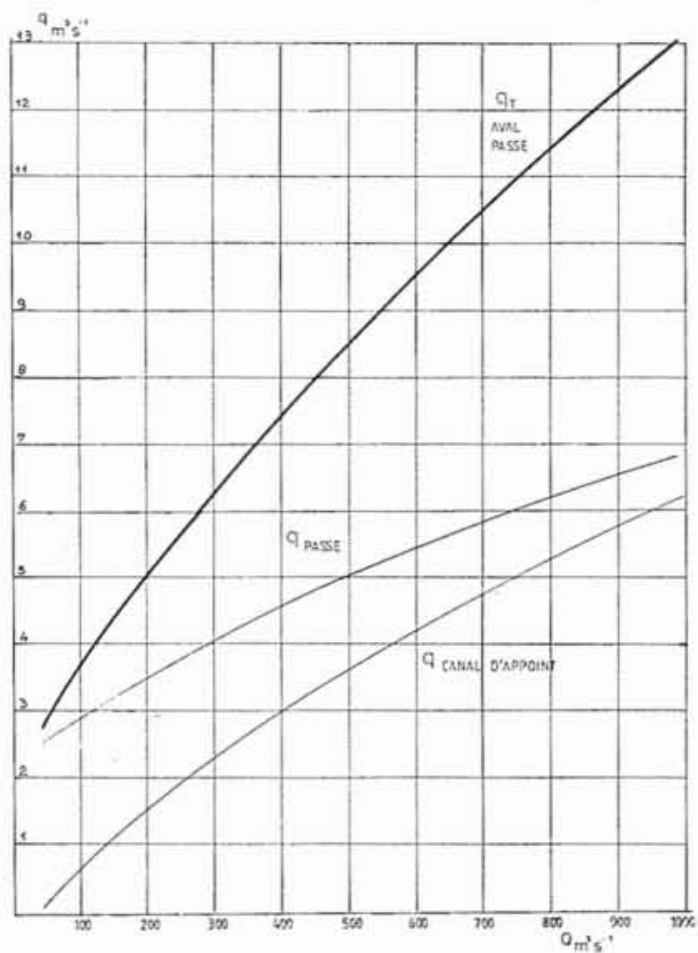

Figure 7. - Débit aux ouvrages.

Photo 3. - Attrait de la passe dans le bief aval pour un débit en Dordogne de $125 \mathrm{~m}^{3} / \mathrm{s}$.

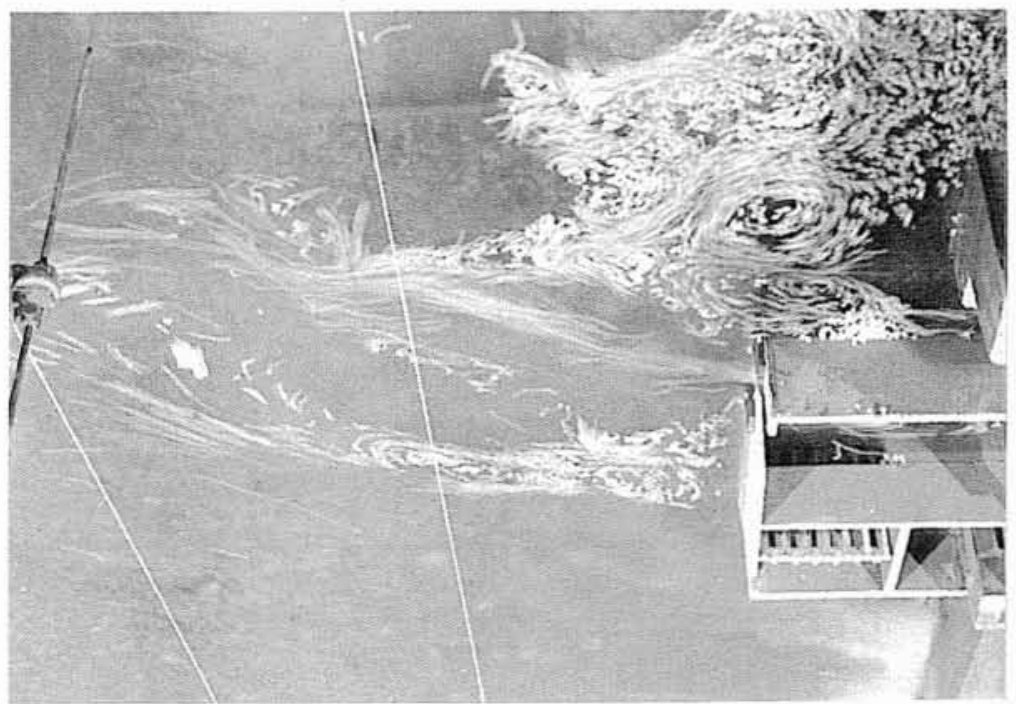

Photo 4. - Écoulement au niveau de la tête amont de l'ouvrage.

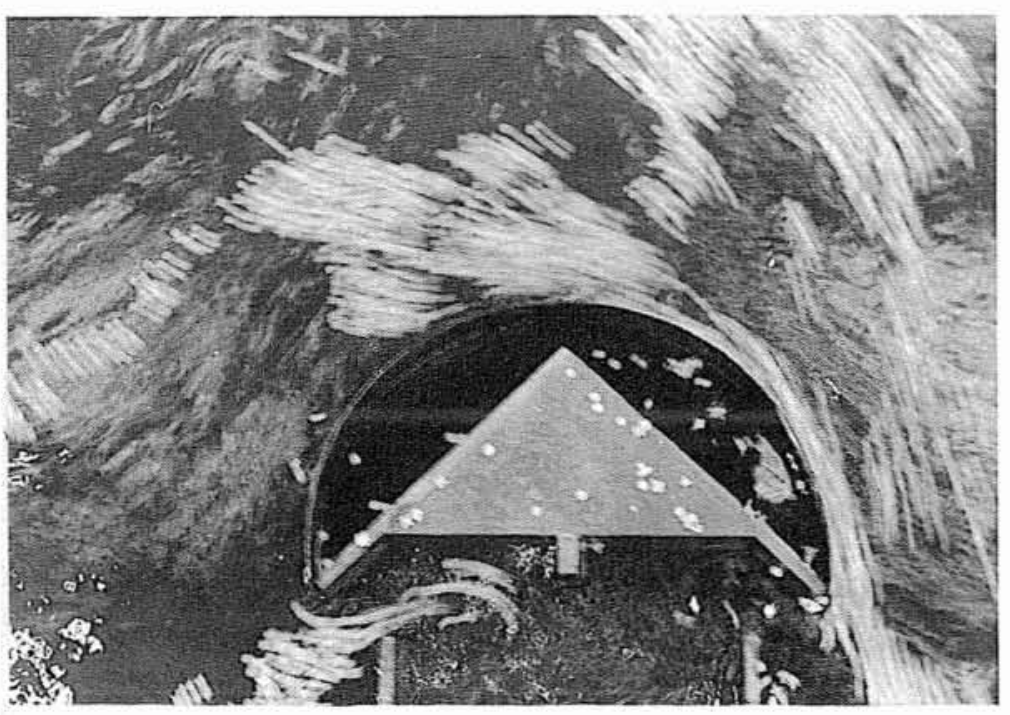

Un canal de 1 mètre de largeur situé dans la pile rive gauche permet l'introduction du débit complémentaire d'attrait. La prise d'eau est protégée par un masque au bordé inférieur calé à la cote 17,82. La dissipation d'énergie du débit d'appoint s'effectue dans trois bassins successifs (Fig. 6). Ce débit est injecté après tranquillisation dans le bassin aval à travers une ouverture de $5 \mathrm{~m} \times$ $3,4 \mathrm{~m}$ équipée de grilles. Ce bassin communique avec le bief aval par une ouverture de 2 mètres de largeur qui constitue l'entrée de la passe. Elle est munie d'une vanne assujettie à un système de réglage maintenant une dénivellation constante entre les cotes des plans d'eau situés de part et d'autre de cette vanne (de l'ordre de 0,25-0,30 m).

Le débit total (passe + appoint) varie de $2,5 \mathrm{~m}^{3} / \mathrm{s}$ environ à plus de $11 \mathrm{~m}^{3} / \mathrm{s}$ pour un débit de $800 \mathrm{~m}^{3} / \mathrm{s}$ en Dordogne (Fig. 7).

Le débit issu de la passe constitue quelles que soient les conditions aval (structures organisées en aval de l'usine, ressaut ou veine ondulée au pied de la chaussée) un jet bien constitué, homogène. La vanne permet d'obtenir des vitesses de $2,50 \mathrm{~m} / \mathrm{s}$ à l'entrée et supérieures à $1 \mathrm{~m} / \mathrm{s}$ à plus de 10 mètres de l'ouvrage. Ce jet s'oriente le plus près possible de l'écoulement principal, c'est-à-dire le long du canal de fuite tant que l'usine fonctionne (Photo 3 ) et en direction de l'écoulement principal lorsque les débits déversés augmentent. L'ouverture du canal de défeuillage (Fig. 8) anihile l'influence d'un vaste courant de retour qui viendrait rabattre le jet sous l'écoulement principal du seuil.

\section{7. Écoulement dans le bief amont et protection de la passe}

L'alimentation de la passe s'effectue par deux prises latérales de 1,30 m de largeur protégées par un avant bec. Initialement, la tête amont était formée d'un dièdre constitué de 2 plans verticaux. Cette configuration a été abandonnée, son efficacité de protection contre les corps dérivants étant faible. L'écoulement dans le bief amont présente fréquemment un angle avec la passe suivant les conditions de fonctionnement de l'usine et l'importance des débits déversés. Les décollements aux angles vifs provoquent de vastes courants de retour qui ramènent les corps flottants le long de la passe jusqu'aux pertuis d'alimentation.

Une tête arrondie de 9 mètres de diamètre a été retenue. Cette forme réduit considérablement les décollements des filets liquides.

Tant que l'usine absorbe la plus grande partie du débit du cours d'eau, il existe une différence de niveau de part et d'autre de la passe. Un très léger courant de retour se forme sur le flanc gauche de la passe et une zone d'eau morte sur le flanc droit. L'alimentation du bassin amont de la passe est correcte et la très légère inégalité aux prises d'eau est réduite au niveau des fentes amont (photo 4). Lorsque tout le débit déverse sur la chaussée, le courant de retour qui se formerait sur le flanc droit de la passe est supprimé par l'ouverture du canal de défeuillage (fig. 8). Le débit est inégalement réparti aux prises d'eau, mais sans conséquences graves. 


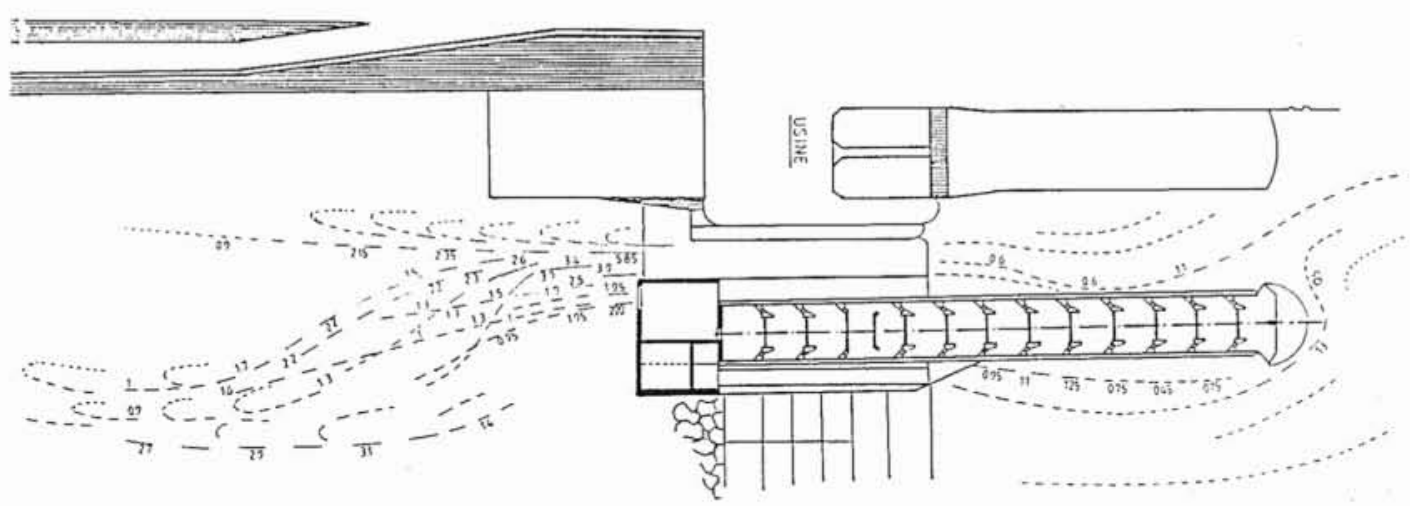

Figure 8. - Cartes de d'écoulement pour un débit en Dordogne de $800 \mathrm{~m}^{3} / \mathrm{s}$.

\section{Conclusions}

La présente étude a permis de caler la passe à poissons et de préciser les caractéristiques de l'écoulement dans les bassins. Le principal intérêt du modèle a été de rendre possible l'optimisation des conditions d'attrait et la définition d'une tête amont assurant une alimentation correcte de l'ouvrage tout en réduisant au maximum la pénétration des corps flottants.

La passe a été construite entre mars et novembre 1984. Une chambre d'observation comportant une paroi vitrée de 6 mètres de hauteur a été installée dans le bassin aval et permet d'observer le passage du poisson au niveau de la fente aval. Les opérations de contrôle ont débuté en mai 1985. Plus de 2000 aloses, 24 saumons, 51 truites de mer et plusieurs milliers d'individus d'autres espèces ont été capturés dans le piège entre mai et décembre 1985. Ces chiffres ne représentent toutefois qu'une partie des migrateurs ayant emprunté la passe.

Les premières observations indiquent que la passe possède une réelle efficacité que les observations et expérimentations des prochaines années permettront de préciser.

\section{Bibliographie}

DUmas, 1979. - Projet de restauration du saumon atlantique dans le bassin de la Dordogne - Saumons, $\mathrm{n}^{\circ} 29$ - p. 6-9.

I.M.F.T., 1982. - Étude sur modèle réduit de la passe à poissons de Belleville - Rapport L.A. 04-005 $\mathrm{n}^{\circ} 391$ - 106 pages.

I.M.F.T., 1983. - Étude sur modèle réduite de la passe à poissons de Bergerac - Rapport L.A. 04-005 n 411 - 109 pp.

LARINIER M., TRAVADE F., 1982. - les poissons migrateurs aux Etats-Unis - Ouvrages de franchissement des barrages Programmes de restauration des populations - EDF-CEMAGREF $-73 \mathrm{p}+$ annexes.
PHILIPPE L., 1897. - Rapport sur les échelles à poissons Ministère de l'Agriculture, Commission des améliorations agricoles et forestières -27 pages.

Pustelnik G., Tinel C., 1985. - Historique, cartographie écologique et évaluation du potentiel d'accueil de la Dordogne pour le saumon atlantique - Colloque franco-québécois sur la Restauration des rivières à saumons - Bergerac, mai-juin 1985.

Roule L., 1929. - Les cours d'eau de notre pays considérés par rapport à la montée du saumon. Rev. Eaux et Forêts - p. 1-15. 
$M^{\text {the }}$ OMBREDANE. - Les passes de Bergerac et de Belleville sont de la même conception. Celle de Bergerac semble très efficace. Mais pour ce qui est de celle de Belleville, les pièges ont permis de constater que très peu d'aloses l'empruntaient. Peut-on expliquer cette différence d'efficacité ?

M. TRAVADE. - Il y a eu un suivi des deux passes: pendant 3 ans à Belleville, 2 ans à Bergerac. Les configurations de ces passes sont en fait peu différentes, mais on ne peut pas parler, pour ce qu'on en sait, de différences d'efficacité, puisque si on observe sous le barrage de Bergerac des accumulations de poissons, on n'a pas pu observer la même chose sous le barrage de Belleville (où seulement 2 aloses ont pu être piégées sur 3 ans d'observation). Il semble y avoir à Belleville une question de blocage de la population avant son arrivée à Belleville.

$M^{\text {te }}$ OMBREDANE. - Si les aloses n'arrivent pas à Belleville, ne fallait-il pas construire des passes sur les barrages situés plus à l'aval sur la Loire dans un premier temps?

Quels sont les critères qui permettent de choisir les priorités dans l'équipement des sites?

M. TANE. - La réponse à vos questions est complexe car les critères qui interviennent dans la décision de construction d'une passe à poissons sont multiples.

Dans les deux cas de Bergerac et Belleville, on se trouve sur des cours d'eau domaniaux oủ la réglementation prévoit l'équipement des seuils par des passes à poissons. Sur les deux sites les équipements s'intégrent à la logique de l'équipement global de l'axe pour les migrateurs. On discute par ailleurs à St-Laurent des Eaux et à Dampierre en Burly pour la mise en place d'un dispositif complémentaire, on est intervenu à Blois par la mise en place d'un réglement d'eau plus adapté.

On a semblé dire que la passe de Belleville ne fonctionnait pas pour les aloses. S'il est vrai qu'il existe des difficultés pour que les aloses parviennent jusqu'à Belleville, on constate qu'en 1983 elles sont montées nettement au-dessus : dans la "branche " Loire jusqu'à Decize et sur l'Allier on en a vu à Vichy.

Si il n'en a pas été piégé cette même année à Belleville c'est que le niveau hydraulique était tel que les poissons pouvaient s'affranchir des échelles : "ils n'ont pas l'esprit assez compliqué pour tenir à passer absolument dans ces échelles quand ils peuvent passer à côté " ". Ces passes sont en effet conçues pour fonctionner dans les situations de débit intermédiaire, où le franchissement direct du barrage est difficile ou impossible. 